\title{
A Newly Designed Resonance Frequency Analysis Device for Dental Implant Stability Detection
}

\author{
Wei-Jen CHANG ${ }^{1}$, Sheng-Yang $\mathrm{LEE}^{2,3}$, Chen-Che $\mathrm{WU}^{2}$, Che-Tong LIN ${ }^{4}$, Yoshimitsu ABIKO ${ }^{5}$, Nobuyuki \\ YAMAMICHI ${ }^{5}$ and Haw-Ming $\mathrm{HUANG}^{4}$ \\ ${ }^{1}$ School of Oral Hygiene, Taipei Medical University, Taipei, Taiwan \\ ${ }^{2}$ School of Dentistry, Taipei Medical University, Taipei, Taiwan \\ ${ }^{3}$ Dental Department of Wan-Fang Hospital, Taipei Medical University, Taipei, Taiwan \\ ${ }^{4}$ Graduate Institute of Oral Sciences, Taipei Medical University, Taipei, Taiwan \\ ${ }^{5}$ Department of Biochemistry, School of Dentistry at Matsudo, Nihon University, Chiba, Japan \\ Corresponding author, Haw-Ming HUANG; E-mail: hhm@tmu.edu.tw
}

Received November 10, 2006/Accepted April 17, 2007

\begin{abstract}
Resonance frequency $(\mathrm{RF})$ analysis technology was used to design a new dental implant stability detector. To calibrate and test the performance of this novel apparatus, in vitro and in vivo models, respectively, were used. The RF values of the test implants detected using our new device and a commercially available analogous device (Osstell) were compared. Further, implant stability status was also detected clinically using our device at 2, 4, 8, and 12 weeks after surgery. A high correlation was demonstrated between the values measured with the two devices $\left(\mathrm{y}=0.31 \mathrm{x}-12.45 ; \mathrm{R}^{2}=0.98, \mathrm{p}<0.05\right)$. In our clinical tests, an initial $\mathrm{RF}$ value above $10.0 \mathrm{kHz}$ indicated that the implant was ready to accept functional loading, while values in the $4.0-10.0 \mathrm{kHz}$ range reflected the need for further osseointegration. In conclusion, these results indicated that our new device might be useful in a clinical setting for evaluating the healing status of a placed implant.
\end{abstract}

Keywords: Resonance frequency, Dental implant, Device

\section{INTRODUCTION}

Presently, development and research pertaining to root form dental implants have advanced to the mature stage. However, the multi-step process of implant treatment is time-consuming and limited in terms of patient acceptance. Although the one-stage implant system provides a partial solution to these problems, the issue of immediate loading is the next logical step to execute ${ }^{1)}$ - and hence the next logical decision to contend with when considering implant stability. Immediate loading refers to a restoration placed in occlusion with the opposing dentition within 48 hours of implant placement ${ }^{2)}$. When the appropriate implant conditions are present, most implants can be immediately loaded. Indeed, many research studies have indicated that the success rate of immediate loading is good $^{3-4)}$. However, primary implant stability is one of the principal factors that governs a dentist's decision on whether a given implant is suitable for accepting immediate loading. Presently, few devices and methods are available for the accurate detection of implant stability immediately after placement.

The use of resonance frequency (RF) to evaluate the extent of bone healing with orthopedic treatment has been studied by many scholars. However, due to the effects of soft tissue, such techniques remain unavailable clinically ${ }^{5}$. In dental research, several recent studies have examined this issue. The results showed that, in principle, RF can be used to monitor the process of osseointegration after dental implant emplacement $t^{6.8)}$.

Osstell (Integration Diagnostics, Göteborgsvängen, Sweden), an RF device based on utilizing harmonic response for monitoring dental implant status, became commercially available in the year 2000. In clinical applications, a transducer is attached to the dental implant fixture and triggered to vibrate by means of sinusoidal waves. The resonance frequency value thus obtained is converted to give an Implant Stability Quotient (ISQ) for analyzing implant stability $^{9-12}$. Recently, this device has been used in implant research to perform the following tasks: compare the success rates between conventional and early loading of implants ${ }^{13-14}$, evaluate the survival rates of transmucosal implants immediately restored with single crowns $^{15}$, monitor the differences between immediate and standard delayed loading of implant $\mathrm{s}^{10-18}$, profile physiological and geometric factors affecting immediate loaded implants ${ }^{19-20}$, and measure stability achieved with one-stage surgical procedures $^{21-22)}$. However, clinical investigation has indicated that temporary attachment, removal, and sterilization of the transducer are time-consuming and far from being cost-effective ${ }^{177}$. In addition, the ISQ value is often influenced by the orientation of the Osstell's L-shaped transducer to the alveolar ridge $^{19,23)}$.

Another type of RF analysis, based on impulse force triggering, has proven useful in terms of detecting dental implant stability in a series of in vivo and in vitro experiments ${ }^{24-26}$. In this study, therefore, the capability of a novel RF detection 
device based on impulse force triggering for monitoring dental implant stability was tested. To reduce measurement operation time, a minimum contact device that did not require additional installation and/or disassembly was designed. To validate the device in terms of implant stability determination, a series of in vitro and in vivo tests was performed. Finally, the standard value for this novel technique, which was to be an indicator of a test implant's readiness for immediate loading, was determined by means of clinical data analysis.

\section{MATERIALS AND METHODS}

\section{Device design}

As shown in Fig. 1, our new device incorporated a minimum contact transducer and an attached handpiece. The handle could be rotated to improve access in the limited space of the oral cavity (Fig. 1(a)). The device consisted of two sections of an electromagnetic coil to provide driving feedback for the demagnetized iron impact head (Fig. 1(b)). When the impulse current passed through the first coil section, the generated electromagnetic field attracted the impact head and drove it to strike against the healing abutment. An impact force of $0.18 \mathrm{~N}$ was thereby delivered, as determined by a pressuresensitive film (Prescale Pressure Series, Fuji Photo Film, Tokyo, Japan). The second section then generated an electromagnetic field in the opposite direction, retracting the impact head to its original position. When the impact head strike the test implant, the resultant vibration was detected via a piezoelectric microphone and the vibration signal sent to a spectrum analyzer (resolution: $50 \mathrm{~Hz}$; Implomates System, Biotech One, Taipei, Taiwan). The specific resonance frequency of the test implant was determined from the relatively highest point with a peak value for vibration amplitude. This device was used throughout the entire research.

\section{In vitro testing}

To validate the experimental device, a series of in vitro tests was carried out. A commercial pure titanium dental implant $(63.75 \times 10 \mathrm{~mm} ; \quad 3 \mathrm{i}$ Innovation, FL) with a 6-mm healing abutment was fixed using a metal clamp stand. RF values for the test implant were recorded while the clamp torque was varied (2 to $10 \mathrm{Nacm}$ in $2 \mathrm{Nacm}$ increments). In addition, the clamping level was altered to expose the implant and alter the vibrational length $(0-8 \mathrm{~mm}$ in $2 \mathrm{~mm}$ steps upward from the base of the implant). Accuracy of the experimental device was tested by comparing the results with those obtained using a conventional excitation method (Osstell). Before RF detection with Osstell, a piezoelectric transducer was connected to the dental implant fixture. Transducer vibration was triggered by $1-\mathrm{V}$ sinusoidal waves in the $5-15 \mathrm{kHz}$ range. The first $\mathrm{RF}$ value detected by the device was used for implant stability analysis.

\section{Animal study}

Five healthy adult beagles (weighing 8-12 kg) were used as test subjects in the animal study. Three months prior to implantation, the mandibular first premolars were bilaterally removed under general (intravenous injection of $0.5 \mathrm{mg} / \mathrm{kg}$ ketamine, followed by intramuscular injection of $25 \mathrm{mg} / \mathrm{kg}$ pentobarbital) and local anesthesia (2\% lidocaine) (Showa Co., Tokyo, Japan). Prior to dental implant surgery, the animals were again anesthetized as described above. Crestal incisions were followed by flap reflection to expose the alveolar crest. The

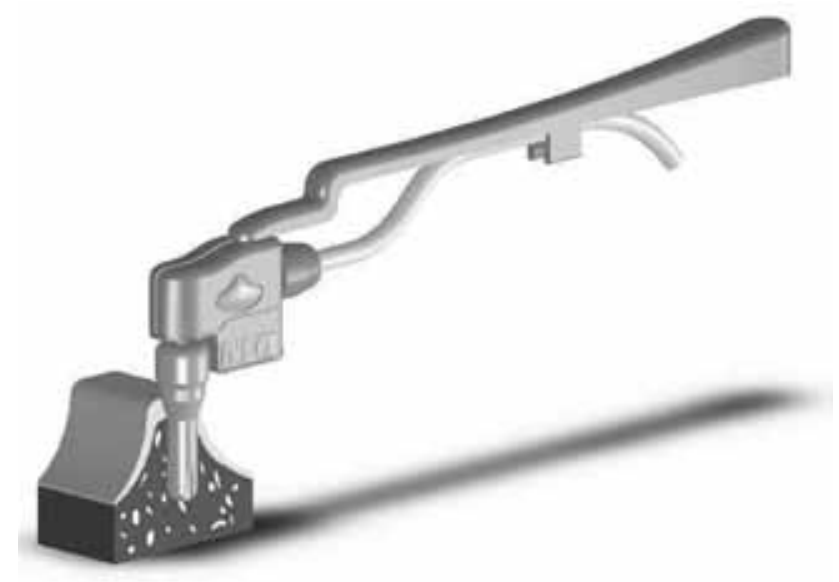

(a)

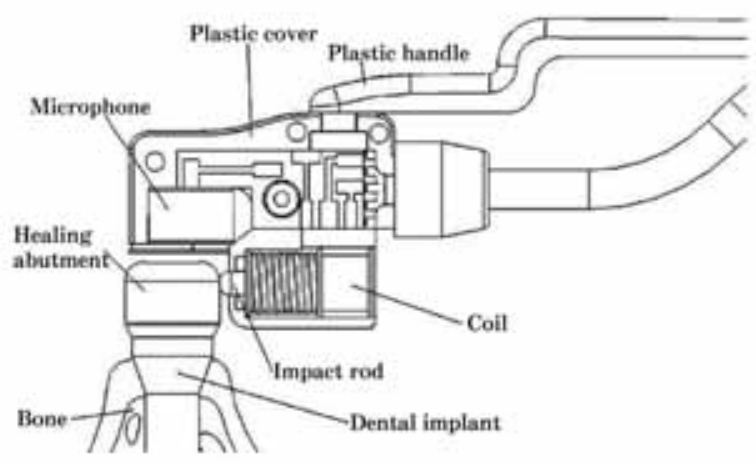

(b)

Fig. 1 Diagrams of the resonance frequency $(\mathrm{RF})$ detector used in this study: (a) Designed as a minimum contact probe, vibration of the test implant was triggered by impact rod; (b) Sagittal section of the device showing electromagnetic driving actuator and non-contacting microphone. 
surgical sites were prepared using the standard procedures specified by the dental implant manufacturer.

Test implants $(63.75 \times 10 \mathrm{~mm}$; 3i Innovation, FL) were placed into the drilled holes at the left first premolar of each animal until their collar margin reached the boundary of the cortical bone. Rightside surgical sites without implant placement were treated as controls. In other words, a total of five implants were used for the entire experiment. After implant placement, the flaps were repositioned and sutured. Profuse cooling with cold normal saline solution was used throughout the surgical procedure. After surgery, the animals were fed a soft diet (Quaker Oats, Peterborough, Ontario, Canada) for the first two weeks, and long-acting penicillin was also administered (Penlong XL, Rogar STB, London, Ontario, Canada) at appropriate intervals during this period.

Immediately after placement of the implants, $\mathrm{RF}$ was measured using both Osstell and our experimental device. Test setup was identical to that used for the in vitro tests above. Measurements were obtained on the implant samples along the buccolingual direction at $0,2,4,8$, and 12 weeks after implant surgery. All samples were subjected to five continuous tests, with results reported as the mean and standard deviation of the RF values. Oneway analysis of variance was used to test statistical differences between the test parameters. The experimental design was approved by the Laboratory Animal Research Committee, College of Oral Medicine, Taipei Medical University.

\section{Clinical data collection}

Data for continuous RF measurement of 11 implants were collected from seven patients (one female and six male patients). All of whom (mean age of 31.4 years, range of $25-48$ years at time of surgery) were fully informed of the study protocol before signing written agreements. The recruitment criteria were: no history of oral disease or dental implant surgery. Edentulous areas were located at the mandibular premolar or first molar (Fig. 2(a)). The 3i implants (3i Innovation, FL) were placed in the mandible according to the manufacturer's guidelines for a onestage procedure (Fig. 2(b)). All the implants were covered with a 4-mm healing abutment to avoid oral fluid contamination. Resonance frequencies were measured using our newly designed apparatus immediately after the implants were placed (week 0) and at weeks 2, 4, 8, and 12 after the implantation surgery (Fig. 2(c)). Detection was performed in the buccolingual direction. After a healing period of 12 weeks, the patients received their prostheses using the classic procedure.

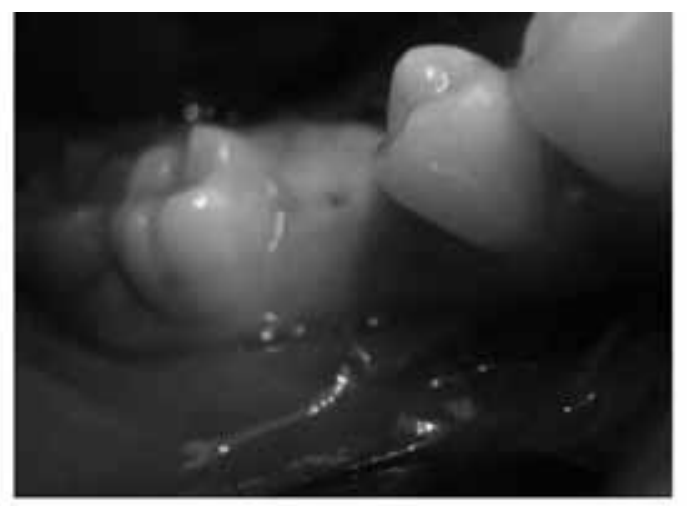

(a)

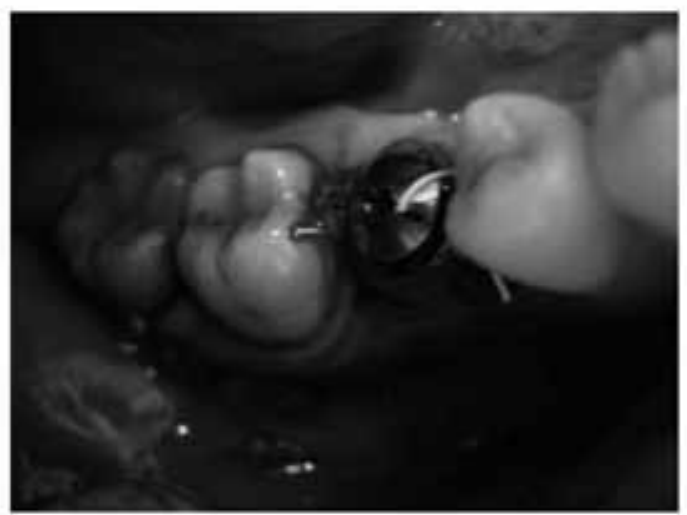

(b)

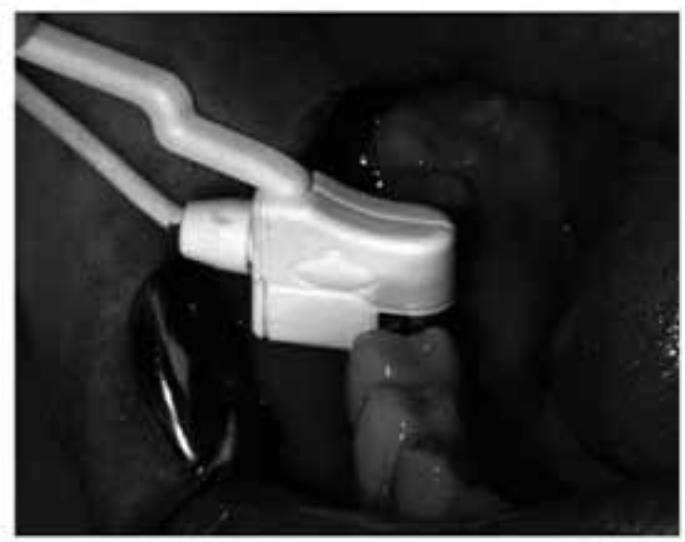

(c)

Fig. 2 Surgical and test procedures: (a) location of edentulous area; (b) general procedure for implant placement; and (c) resonance frequencies of test implants detected using the new device.

\section{RESULTS}

Table 1 shows the RF and ISQ values, as measured in vitro using our novel device and Osstell respectively, for a dental implant with various clamping torques. The mean experimental $\mathrm{RF}$ value increased from $14.76 \pm 0.02$ to $15.11 \pm 0.02 \mathrm{kHz}$ in the torque range $2-10$ Na cm. Similarly, under the same test 
Table 1 Comparison of implant measurement data generated by Osstell and new RFA device for different clamping torques

\begin{tabular}{lccccccc}
\hline & Torque $(\mathrm{N} n \mathrm{~cm})$ & 2 & 4 & 6 & 8 & 10 \\
Device & & & & & & & \\
\hline Osstell (ISQ) & $85.67 \pm 0.58$ & $88.00 \pm 0.00$ & $88.00 \pm 0.00$ & $89.00 \pm 0.00$ & $89.00 \pm 0.00$ \\
Newly designed device $(\mathrm{kHz})$ & $14.76 \pm 0.02$ & $14.90 \pm 0.00$ & $15.01 \pm 0.02$ & $15.10 \pm 0.00$ & $15.11 \pm 0.02$ \\
\hline
\end{tabular}

Data are presented as mean $\pm \mathrm{SD}$.

Table 2 Comparison of implant measurement data from Osstell and new RFA device for different clamping levels

\begin{tabular}{|c|c|c|c|c|c|}
\hline $\begin{array}{l}\text { Exposed height }(\mathrm{mm}) \\
\text { Device }\end{array}$ & 0 & 2 & 4 & 6 & 8 \\
\hline Osstell (ISQ) & $88.00 \pm 0.00$ & $77.67 \pm 0.00$ & $70.00 \pm 0.00$ & $64.67 \pm 0.58$ & $56.33 \pm 1.53$ \\
\hline Newly designed device $(\mathrm{kHz})$ & $16.10 \pm 0.00$ & $12.31 \pm 0.02$ & $9.16 \pm 0.02$ & $7.01 \pm 0.02$ & $5.29 \pm 0.02$ \\
\hline
\end{tabular}

Data are presented as mean $\pm \mathrm{SD}$.

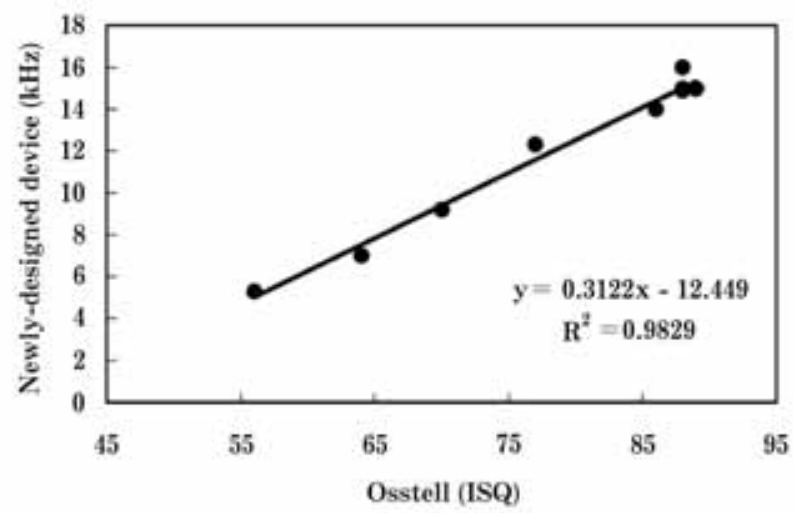

Fig. 3 Relationship of data measured in vitro for the two devices.

conditions using the Osstell device, the ISQ value also increased from $85.67 \pm 0.58$ to $89.00 \pm 0.00$. However, no differences in ISQ value were detected when clamping torque was increased from 4 to $6 \mathrm{Na} \mathrm{cm}$ (ISQ =88), and from 8 to $10 \mathrm{Nacm} \quad(\mathrm{ISQ}=89)$. Table 2 lists the measurement data for the various clamping levels. When the exposed height of the test implant was increased from 0 to $8 \mathrm{~mm}$, the mean experimental $R F$ value decreased from $16.10 \pm 0.00$ to $5.29 \pm 0.02 \mathrm{kHz}$. Similarly, the measured ISQ value decreased from $88.00 \pm 0.00$ to $56.33 \pm 1.53$ for the same test conditions. Replotting the measurement data from Tables 1 and 2 in Fig. 3, a linear correlation was obtained between the $R F$ values of our newly designed device and ISQ values derived from Osstell $(\mathrm{r}=0.991 ; \mathrm{P}<0.01)$.

All animals used in the in vivo study remained in excellent health throughout the course of the experiment. The in vivo data, in the form of continuous measurements obtained using our novel device, are plotted in Fig. 4(a). There was a mean significant decrease of $1.45 \mathrm{kHz}$ between initial placement and two weeks post surgery $(\mathrm{P}<0.05)$, but a mean significant increase of $2.45 \mathrm{kHz}$ between weeks 2 and $12(\mathrm{P}<0.01)$. More importantly, when the same implants were tested using the Osstell device, no significant differences were detected between the ISQ values across the experimental period (Fig. 4(b)).

Figure 5 shows the results of the clinical investigation, whereby test implants were divided into three groups. In Group I, the initial RF values were above $9 \mathrm{kHz}$, remaining high and plateauing at $11 \mathrm{kHz}$ or more by week 12 . In Group II, the initial RF values of the implants ranged between 3.58 and $5.3 \mathrm{kHz}$. These implants remained in excellent health throughout the course of the experiment and completed the osseointegration process. By week 12 , the RF values for all the Group II implants were above $9.8 \mathrm{kHz}$. In Group III, one implant with an initial RF value of $3.56 \mathrm{kHz}$ did not demonstrate an increasing trend in $\mathrm{RF}$ value after the first two weeks of healing. Osseointegration of the implant failed, and by week 12 it loosened with a finial $\mathrm{RF}$ value of $3.85 \mathrm{kHz}$. Our short-term results demonstrated a $91 \%$ success rate during the first 12 weeks.

To assess the relationship between the $\mathrm{RF}$ values of immediate and delayed loaded implants in this study, the resonance frequency increase ratio (RFIR) was defined as the ratio between the initial $R F$ value and the analogous value at week 4 . The mean RFIR of Group II implants (1.98 \pm 0.31) was significantly higher than that of Group I analog (1.09 \pm 0.11 ; $\mathrm{P}<0.005)$. The plot in Fig. 6 revealed a linear relationship between the RFIR of each test implant and its initial $R F\left(y=-0.126 x+2.50, \quad R^{2}=0.811\right.$, $\mathrm{p}<0.05)$. 

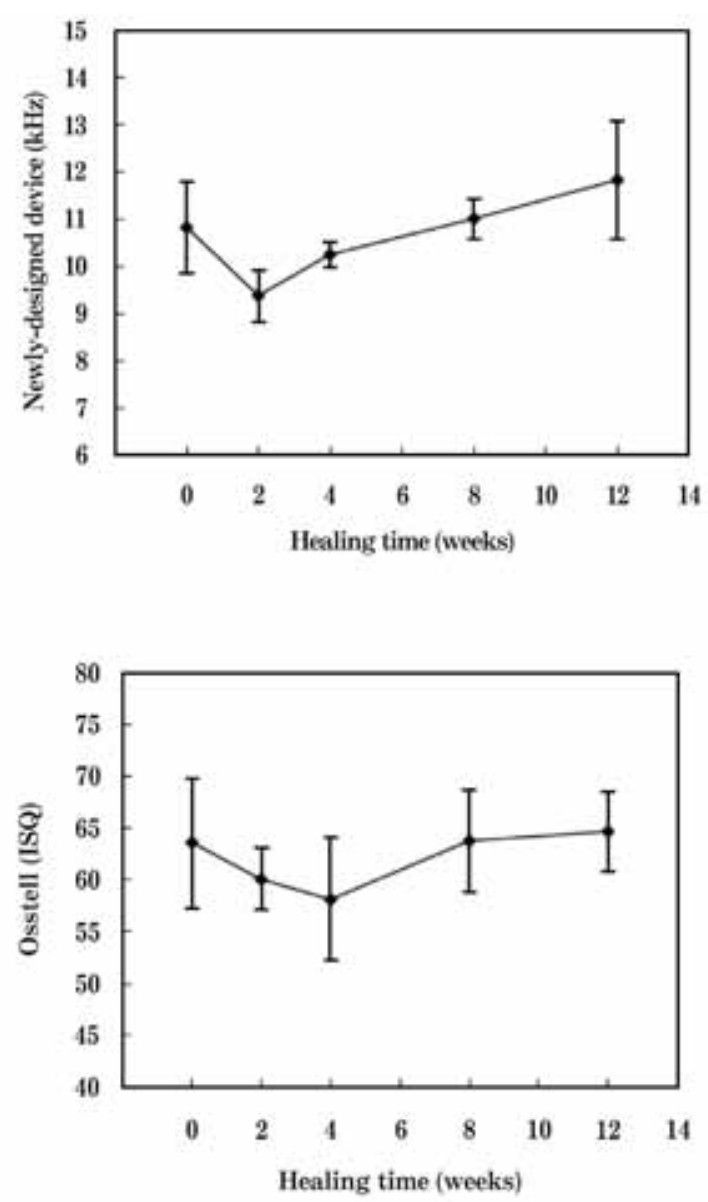

(b)

Fig. 4 Healing curves generated using RF values obtained from test implants in experimental animals with (a) experimental device and (b) Osstell. The data are presented as mean $\pm \mathrm{SD}$.

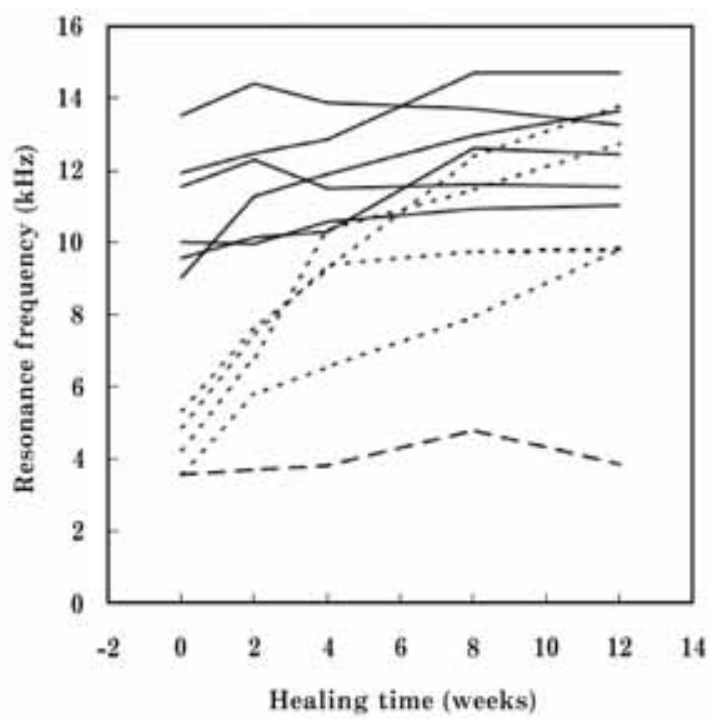

Fig. 5 RF healing curves from test implants in patients during the 12 weeks post implantation. Solid, short-dashed, and long-dashed lines denote Groups I, II, and III (initial RF values $>9,3.5_{-} 6$, and $<4$ $\mathrm{kHz}$, respectively).

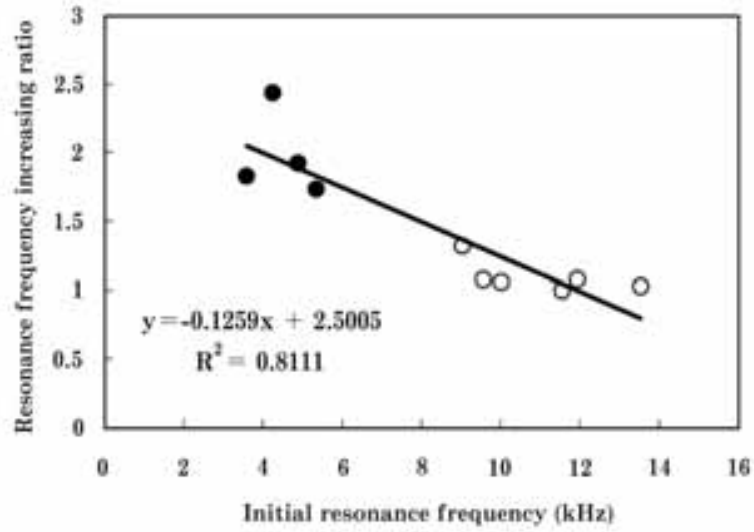

Fig. 6 Relationship between the initial $R F$ value and $R F$ increase ratio of the successful implants at four weeks post surgery. Open and closed circles denote Group I and Group II implants respectively.

\section{DISCUSSION}

Osstell was the first commercially available RF device to test dental implant stability, and the efficacy of this method for monitoring implant status has been proven $^{10-12}$. However, it is somewhat inconvenient and time-consuming ${ }^{177}$, and it may even affect the implant-bone interface at the early healing stage. This is because the Osstell transducer must be screwed into the test implant with a torque of 10 Na cm, almost half the force used to place an implant. In this light, the mechanical effect of disassembling the healing abutment on the interface in the early stages of osseointegration should be taken into consideration. Against this background, our novel transducer was designed as a minimum contact device with no torque force required during transducer application.

In the development of a medical device, accuracy of the measuring instrument should be an utmost priority for the engineers involved. To validate the effectiveness of our newly designed apparatus, in vitro tests were performed with a view to reducing the physiological effects of measurement. According to previous studies, resonance frequency was affected by bone-implant contact percentage and height of the exposed implant above the marginal bone ${ }^{6,24,25)}$. In this study, therefore, both factors were simulated and tested in vitro as in our previous work ${ }^{24)}$. As shown in Fig. 3, a close relationship was demonstrated for the data obtained using Osstell and our device. Thus, it seemed reasonable to suggest that our novel device may be used to monitor changes in implant $\mathrm{RF}$ with various boundary conditions, while offering significant functional advantages and without adversely affecting the healing process.

In the present study, the mean $R F$ of the implants placed in animals decreased during the first 
two weeks and then increased progressively with time (Fig. 4). These results were consistent with other animal $\mathrm{RFA}^{14,26)}$ and reverse torque studies ${ }^{16,27)}$. This initial reduction in stability might correspond to the remodeling and neo-apposition phases of the necrotized bone ${ }^{16)}$. However, the stability-return phenomenon was not borne out in our clinical data (Fig. 5). In fact, demonstrating this phenomenon using resonance frequency analysis (RFA) remains controversial. Although a tendency for ISQ to decrease in the initial stage followed by a stable increase has been demonstrated in some clinical studies $^{14,18-19,28)}$, many RFA-based investigations have not confirmed this phenomenon ${ }^{16,23}$. After testing 276 immediate loaded implants using RFA, Balshi et al. suggested that bone types II and III were more consistent in term of stability return, while bone types I and IV were not ${ }^{19}$.

Primary stability, a function of bone quality, implant design, and surgical procedure, is one of the important factors that influences the survival rate of implants ${ }^{17,2027)}$. It has been reported that primary stability can serve as a useful predictor of osseointegration $^{16)}$. Further, it has been demonstrated that good primary implant stability, as measured by RFA, did not increase significantly during the osseointegration period ${ }^{15,16,29)}$. In this study, it was found that implants with higher stability indeed had relatively stable $\mathrm{RF}$ during the whole healing period with a mean RFIR of $1.09 \pm 0.112$ (Figs. 5 and 6). As constant or slightly increased $R F$ values for immediate loaded implants during the first 4-6 weeks have been reported ${ }^{16)}$, it seemed reasonable to suggest that for implants with initial $R F$ values exceeding $10 \mathrm{kHz}$ (as measured by our device), these implants are ready for immediate loading.

A number of researches have proposed that where the initial ISQ value is above 60, the implant is ready for immediate loading ${ }^{17-19)}$. However, for implants with ISQ values below 40, this should serve as a warning of early failure ${ }^{18)}$. Interestingly, this proposed functional threshold of ISQ 60 is equivalent to an $\mathrm{RF}$ of $6.10 \mathrm{kHz}^{18)}$, much lower than our experimentally determined limit of $10 \mathrm{kHz}$. A negative relationship has been reported between $\mathrm{RF}$ value and effective vibrational length above bone level ${ }^{8,16,24-25)}$. Since the Osstell transducer is an Lshaped device that must be attached to the top of the test implant, the effective vibrational length of test implant would thus be greater than that tested using our device. Accordingly, to compare and exchange measurement data generated using the Osstell system (ISQ) and the analogous information from our own device (in $\mathrm{kHz}$ ), a transfer function was derived.

As shown in Fig. 3, the relationship between the two parameters was linear with a ratio of 0.3 . As the relationship formula in Fig. 3 was obtained from in vitro tests, the constant in the formula should be modified based on our clinical data. After substituting $10 \mathrm{kHz}$ and 60 ISQ for $\mathrm{y}$ and $\mathrm{x}$ in Fig. 3 respectively, a new transfer function was derived: $\mathrm{y}=0.3 \mathrm{x}-$ 8 , where $\mathrm{y}$ and $\mathrm{x}$ are $\mathrm{kHz}$ and ISQ values respectively. Based on this function, the early failure threshold of ISQ 40 for Osstell was $4 \mathrm{kHz}$ using our novel device. From Fig. 5, the initial and final RF values of the failed implant $(3.56 \mathrm{kHz}$ and $3.85 \mathrm{kHz}$, respectively) fit the hypothesis well.

The initial RF values of our test implants varied (Fig. 5), probably reflecting the marked interindividual variations in distributions of cortical and trabecular bone, as well as differences between various locations within bone ${ }^{30)}$. After analyzing the RFA data of 120 one-stage implants, Zix et al. suggested that a single RFA measurement for an implant at a given time point could not qualify being a full predictor of its future performance ${ }^{22}$. Repeated measurements of an implant over a period would provide a more credible prognosis of its future status. In this connection, Glauser et al. suggested that low RFA levels after one and two months implied risk for future failure ${ }^{18)}$.

In the current study, implants with lower initial $R F$ values revealed significantly higher RFIR values (Fig. 6). At this juncture, it must be clarified that RFIR was defined in this study for early diagnosis but not for determining the optimal time point for functional loading. However, it seemed reasonable to suggest that the time for functional loading of these implants is when their $R F$ value plateaus or exceeds $9 \mathrm{kHz}$. Nedir et al. also reported that implants with lower initial ISQ values demonstrated a larger ISQ increase during the healing period ${ }^{17)}$. In contrast, higher initial stability was associated with a more stable ISQ value. In addition, only the implant with highest stability revealed stability decrease during the first four weeks before remaining stable. These results substantially agreed with our own findings.

Based on the results obtained in the present study, it was therefore reasonable to suggest that our novel $\mathrm{RF}$ detection device was suitable for evaluating the integrity of bone union during the osseointegration process. At the same time, our novel device provided functional superiority without adversely affecting the restorative process. Amongst which, the greatest advantage of this new device was its ergonomic superiority. Thus, we proposed that our newly designed RFA apparatus had the potential of delivering substantial benefits to advanced experiments in the future, as well as holding out significant promise for future clinical use.

\section{REFERENCES}

1) Raghoebar GM, Schoen P, Meijer HJA, Stellingsma 
K, Vissink A. Early loading of endosseous implants in the augmented maxilla: a 1-year prospective study. Clin Oral Impl Res 2003; 14: 697-702.

2) Cochran DL, Morton D, Weber HP. Consensus statements and recommended clinical procedures regarding loading protocols for endosseous dental implants. Int J Oral Maxillofac Impl 2004; 19: 109-113

3) Horiuchi K, Uchida H, Yamamoto K, Sugimura M. Immediate loading of Bränemark system implants following placement in edentulous patients: a clinical report. Int J Oral Maxillofac Impl 2000; 15: 824-830.

4) Andersen E, Haanaes HR, Knutsen BM. Immediate loading of single-tooth ITI implants in the anterior maxilla: a prospective 5-year pilot study. Clin Oral Impl Res 2002; 13: 281-287.

5) Nokes LD. The use of low-frequency vibration measurement in orthopaedics. Proc Instn Mech Engrs, Part H: J Eng Med 1999; 213: 271-290.

6) Meredith N, Alleyne D, Cawley P. Quantitative determination of the stability of the implant-tissue interface using resonance frequency analysis. Clin Oral Impl Res 1996; 7: 261-267.

7) Meredith N. Assessment of implant stability as a prognostic determinant. Int $\mathrm{J}$ Prosthodont 1998; 11: 491-501.

8) Meredith N, Shagaldi F, Alleyne D, Sennerby L, Cawley P. The application of resonance frequency measurements to study the stability of titanium implants during healing in the rabbit tibia. Clin Oral Impl Res 1997; 8: 234-243.

9) Meredith N. A review of nondestructive test methods and their application to measure the stability and osseointegration of bone anchored endosseous implants. Crit Rev Biomed Eng 1998; 26: 275-291.

10) Cawley P, Pavlakovic B, Alleyne DN, George R, Back $\mathrm{T}$, Meredith N. The design of a vibration transducer to monitor the integrity of dental implants. Proc Instn Mech Engrs, Part H: J Eng Med 1998; 212: 265272 .

11) Rasmusson L, Meredith N, Cho IH, Sennerby L. The influence of simultaneous versus delayed placement on the stability of titanium implants in onlay bone grafts: A histologic and biomechanic study in the rabbit. Int J Oral Maxillofac Surg 1999; 28: 224-231.

12) Barewal RM, Oates TW, Meredith N, Cochran DL. Resonance frequency measurement of implant stability in vivo on implants with a sandblasted and acidetched surface. Int J Oral Maxillofac Implants 2003; 18: 641-651.

13) Payne AGT, Tawse-Smith A, Duncan WD, Kumara R. Conventional and early loading of unsplinted ITI implants supporting mandibular overdentures: twoyear results of a prospective randomized clinical trial. Clin Oral Impl Res 2002; 13: 603-609.

14) De Smet E, Jaecques S, Vandamme K, Vander Sloten J, Naert I. Positive effect of early loading on implant stability in the bi-cortical guinea-pig model. Clin Oral Impl Res 2005; 16: 402-407.

15) Cornelini R, Cangini F, Covani U, Barone A, Buser D. Immediate restoration of single-tooth implants in mandibular molar sites: A 12-month preliminary report. Int J Oral Maxillofac Implants 2004; 19: 855860 .

16) Bischof M, Nedir R, Szmukler-Moncler S, Bernard JP, Samson J. Implant stability measurement of delayed and immediately loaded implants during healing. A clinical resonance-frequency analysis study with sandblasted-and-etched ITI implants. Clin Oral Impl Res 2004; 15: 529-539.

17) Nedir R, Bischof M, Szmukler-Moncler S, Bernard JP, Samson J. Predicting osseointegration by means of implant primary stability. A resonance-frequency analysis study with delayed and immediately loaded ITI SLA implants. Clin Oral Impl Res 2004; 15: 520528 .

18) Glauser R, Sennerby L, Meredith N, Ree A, Lundgren AK, Gottlow J, Hammerle CHF. Resonance frequency analysis of implants subjected to immediate or early functional occlusal loading. Successful vs. failing implants. Clin Oral Impl Res 2004, 15: 428434.

19) Balshi SF, Allen FD, Wolfinger GJ, Balshi TJ. A resonance frequency analysis assessment of maxillary and mandibular immediately loaded implants. Int $\mathrm{J}$ Oral Maxillofac Implants 2005; 20: 584-594.

20) Akkocaoglu M, Uysal S, Tekdemir I, Akca K, Cehreli MC. Implant design and intraosseous stability of immediately placed implants: A human cadaver study. Clin Oral Impl Res 2005; 16: 202-209.

21) Monov G, Fuerst G, Tepper G, Watzak G, Zechner W, Watzek G. The effect of platelet-rich plasma upon implant stability measured by resonance frequency analysis in the lower anterior mandibles: A pilot study. Clin Oral Impl Res 2005; 16: 461-465.

22) Zix J, Kessler-Liechti G, Mericske-Stern R. Stability measurements of 1-stage implants in the maxilla by means of resonance frequency analysis: A pilot study. Int J Oral Maxillofac Implants 2005; 20: 747-752.

23) Kramer FJ, Dempf R, Bremer B. Efficacy of dental implants placed into fibula-free flaps for orofacial reconstruction. Clin Oral Impl Res 2005; 16: 80-88.

24) Huang HM, Pan LC, Lee SY, Chiu CL, Fan KH, Ho KN. Assessing the implant/bone interface by using natural frequency analysis. Oral Surg Oral Med Oral Pathol Oral Radiol Endod 2000; 90: 285-291.

25) Huang HM, Lee SY, Yeh CY, Lin CT. Resonance frequency assessment of dental implant stability with various bone qualities: a numerical approach. Clin Oral Impl Res 2002; 13: 65-74.

26) Huang HM, Chiu CL, Yeh CY, Lin CT, Lin LH, Lin LH, Lee SY. Early detection of implant healing process using resonance frequency analysis. Clin Oral Impl Res 2003; 14: 437-443.

27) Baker D, London RM, O'Neal R. Rate of pull-out strength gain of dual-etched titanium implants: a comparative study in rabbits. Int $\mathrm{J}$ Oral Maxillofacial Implants 1999; 14: 722-728.

28) Monov G, Fuerst G, Tepper G, Watzak G, Zechner W, Watzek G. The effect of platelet-rich plasma upon implant stability measured by resonance frequency analysis in the lower anterior mandibles. A pilot study. Clin Oral Impl Res 2005; 16: 461-465.

29) Friberg B, Sennerby L, Linden B, Grondahl K, Lekholm U. Stability measurements of one-stage Branemark implants during healing in mandibles. A clinical resonance frequency analysis study. Int $\mathrm{J}$ Oral Maxillofac Surg 1999; 28: 266-272.

30) Fanuscu MI, Chang TL. Three-dimensional morphometric analysis of human cadaver bone: microstructural data from maxilla and mandible. Clin Oral Impl Res 2004; 15: 213-218. 